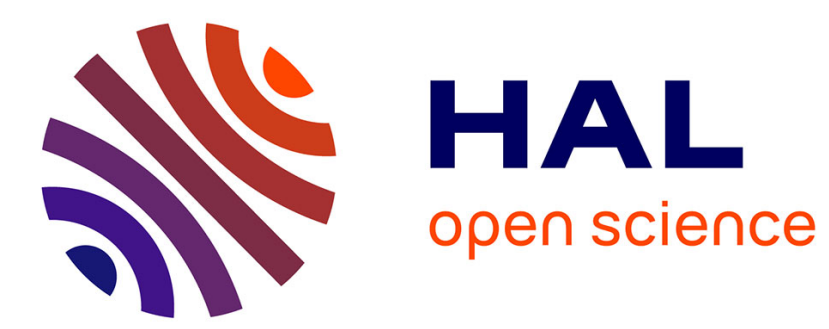

\title{
Des techniques de soi ambivalentes
}

Alexandre Coutant

\section{To cite this version:}

Alexandre Coutant. Des techniques de soi ambivalentes. Hermès, La Revue - Cognition, communication, politique, 2011, 59, pp.53-58. hal-00843310

\section{HAL Id: hal-00843310 https://hal.science/hal-00843310}

Submitted on 12 Jul 2013

HAL is a multi-disciplinary open access archive for the deposit and dissemination of scientific research documents, whether they are published or not. The documents may come from teaching and research institutions in France or abroad, or from public or private research centers.
L'archive ouverte pluridisciplinaire HAL, est destinée au dépôt et à la diffusion de documents scientifiques de niveau recherche, publiés ou non, émanant des établissements d'enseignement et de recherche français ou étrangers, des laboratoires publics ou privés. 


\section{Des techniques de soi ambivalentes}

Alexandre Coutant

Université de Franche-Comté - équipe Objets et Usages Numériques, laboratoire LASELDI

(EA2281). Chercheur associé à l'ISCC.

«Si nous considérons l'ensemble des machines que notre civilisation livre à l'usage de l'individu, nous verrons que leurs caractères techniques sont oblitérés et dissimulés par une impénétrable rhétorique, recouverts d'une mythologie et d'une magie collective qu'on arrive à peine à élucider ou démystifier. Les machines modernes utilisées dans la vie quotidienne sont pour la plupart des instruments de flatterie. Il existe une sophistique de la présentation qui consiste à donner une tournure magique à l'être technique, pour endormir les puissances actives de l'individu et l'amener à un état hypnotique dans lequel il goûte le plaisir de commander à une foule d'esclaves mécaniques, souvent assez peu diligents et peu fidèles, mais toujours flatteurs. »

In SIMONDON, G. (2007). L'individuation psychique et collective : à la lumière des notions de Forme, Information, Potentiel et Métastabilité.

Michel Foucault a établi, dans son cycle de conférences à l'université du Vermont en 1982, l'archéologie de ce qu'il appelle les techniques de soi. Ce terme concernait alors uniquement les techniques intellectuelles favorisant l'émergence de types de subjectivité, mais Foucault l'a rapidement associé à un ensemble d'études concernant «les arts de soi-même » où il insistait notamment sur l'écriture de soi (regroupées dans Foucault, 2001). Il définit alors les techniques de soi comme permettant « aux individus d'effectuer, seuls ou avec l'aide d'autres, un certain nombre d'opérations sur leur corps et leur âme, leurs pensées, leurs conduites, leur mode d'être; de se transformer afin d'atteindre un certain état de bonheur, de pureté, de sagesse, de perfection ou d'immoralité » (p. 1604). La sociologie de l'individu a depuis repris cette démarche en s'attachant à rendre compte de comment le processus identitaire se fonde sur un vaste ensemble de supports (Martuccelli, 2002). Ils peuvent ainsi être relationnels (communautés d'appartenance, famille, amis), intellectuels (psychanalyse, développement personnel) ou même physiques (journal intime, miroir). Ces travaux soulignent à quel point l'identité ne saurait être perçue autrement que comme une co-construction négociée entre les interactants (Goffman, 1974), humains ou non humains (Kaufmann, 2005).

\section{Les réseaux socionumériques, une apparente technique de soi}

À bien des égards, les réseaux socionumériques (Rsn) évoquent les outils analysés par Foucault. On y retrouve les mêmes détails du quotidien, les humeurs consignées et les faits ayant ponctué la journée. Ainsi, à propos des hypomnemata ${ }^{i}$, cette description qui pourrait être 
appliquée à Facebook: «on y consignait des citations, des fragments d'ouvrages, des exemples et des actions dont on avait été témoin ou dont on avait lu le récit, des réflexions ou des raisonnements qu'on avait entendus ou qui étaient venus à l'esprit. Ils constituaient une mémoire matérielle des choses lues, entendues ou pensées » (Foucault, 2001, p. 1237). La vocation des techniques de soi à « amasser des contenus disparates pour en faire une unité » (p. 1241), ne peut manquer non plus d'évoquer les profils des utilisateurs, amassant statuts, liens, vidéos, photos, tests, affirmation de goûts dans multiples domaines pour constituer une présentation de soi fine. On retrouve sur les Rsn la même narration de soi permettant de s'approprier ces contenus disparates pour les transformer «en forces et en sang », en corps de notre identité : «le rôle de l'écriture est de constituer, avec tout ce que la lecture a constitué, un « corps »» (p. 1241). La dimension interactive de la construction des profils des Rsn y est déjà soulignée, à travers les exercices épistolaires recommandés par Sénèque à ses élèves. Il y est question de se découvrir dans l'échange avec l'autre : «le travail que la lettre opère sur le destinataire, mais qui est aussi effectué sur le scripteur par la lettre même qu'il envoie, implique donc une «introspection»; mais il faut comprendre celle-ci moins comme un déchiffrement de soi par soi que comme une ouverture qu'on donne à l'autre sur soi-même » (p. 1245). De la même manière, l'écriture de soi sur les profils vaut moins pour le déclaratif en lui-même que pour les réactions attendues de nos audiences, qu'elles restent à l'état de commentaires sans suite ou qu'elles provoquent de longs échanges.

On retrouve enfin dans les contenus les mêmes insistances sur le corps et le quotidien comme objets constitutifs de l'écriture du rapport à soi : « les interférences de l'âme et du corps (les impressions plutôt que les actions) et les activités du loisir (plutôt que les événements extérieurs)»(p. 1245). Le renouvellement permanent des statuts pour témoigner des nouveaux détails du quotidien et la multiplication des activités narcissiques ${ }^{\mathrm{ii}}$ illustrent cet ancrage charnel et ordinaire de la culture de soi (Coutant, Stenger, 2010b).

\section{Des caractéristiques qui s'éloignent du soin de soi}

Cependant, les Rsn possèdent des particularités qui les distinguent très nettement du « soin de soi ». Si les différentes techniques passées en revue par Foucault relèvent d'actions volontaires de la part des individus, le déploiement du soi sur les Rsn s'avère bien moins maîtrisé et consciemment orchestré. Par ailleurs, si les techniques de soi classiques envisagent le partage des résultats de ses activités avec d'autres, elles ne mettent pas en place un système absolument interactionniste comme le font les Rsn. 


\section{Une maîtrise individuelle du soi limitée}

Les techniques de soi se donnent pour objectif une meilleure administration de soi, passant notamment par la capacitation à effectuer une sélection des faits vécus dignes d'intérêt. Elles reflètent des visions plus ou moins rationalisantes du sujet, des inclinaisons morales diverses et des conceptions plus ou moins immanentistes du soi selon les périodes. Il n'en demeure pas moins que, dans tous les cas, elles visent à donner au sujet un recul réflexif sur lui-même et à lui procurer la capacité d'évaluer et sélectionner des éléments de son expérience. La logique de flux d'activités mise en place par les Rsn ne relève en rien de cette volonté (Coutant, Stenger, 2010a). Au contraire, ces derniers poussent à abandonner la réflexivité et les tentatives de stabilisation du soi (même si elles demeurent incomplètes et temporaires) pour se noyer dans le flux sans cesse renouvelé de traces de soi et des autres. Pour l'individu souhaitant exister sur ces réseaux, il faut renouveler sans cesse sa participation, quitte à accorder peu d'attention aux traces passées ${ }^{\text {iii }}$. Cette caractéristique diffère totalement des hypomnemata destinés au contraire à capter le déjà-dit: «ils offraient ainsi un trésor accumulé à la relecture et à la médiation ultérieures. Ils formaient ainsi une matière première pour la rédaction de traités plus systématiques, dans lesquels on donnait les arguments et moyens pour lutter contre tel défaut (comme la colère, l'envie, le bavardage, la flatterie) ou pour surmonter telle circonstance difficile (un deuil, un exil, la ruine, la disgrâce) » (Foucault, 2001, p. 1237).

Plusieurs travaux s'intéressant à l'identité sur les Rsn aboutissent au même constat d'une perte en matière de gestion de soi encouragée par ces plateformes (Coutant, Stenger, 2010a ; Georges \& al, 2009). Les Rsn mettent en exergue ce que Kaufmann nomme des identités ICO (immédiates, contextualisées et opératoires). Il s'agit d'éléments identitaires dynamiques qui s'activent sur le moment et conviennent à un contexte donné, mais qui sont oubliés aussi vite. Les identités ICO permettent de s'adapter au contexte immédiat mais servent peu à l'autre aspect de la dynamique identitaire qui cherche la stabilisation et la pérennisation d'une image du moi. Les techniques de soi doivent permettre d'y distinguer le mémorable de l'insignifiant, ce que ne font pas les Rsn en plaçant toutes les informations au même niveau dans le flux. Ils provoquent une sorte de fuite en avant de la construction identitaire car les activités narcissiques qui y sont poursuivies ne cessent de disparaître dans le flux là où elles devraient fournir des îlots, même temporaires, de stabilité. Les Rsn se rapprochent alors davantage de la stultitia: «la stultitia se définit par l'agitation de l'esprit, l'instabilité de l'attention, le changement des opinions et des volontés, et par conséquent la fragilité devant tous les 
événements qui peuvent se produire ; elle se caractérise aussi par le fait qu'elle tourne l'esprit vers l'avenir, le rend curieux de nouveautés et l'empêche de se donner un point fixe dans la possession d'une vérité acquise. L'écriture des hypomnemata s'oppose à cet éparpillement en fixant des éléments acquis et en constituant en quelque sorte «du passé », vers lequel il est toujours possible de faire retour et retraite $»$ (Foucault, 2001, p. 1239).

Dans ce contexte, il devient urgent d'opposer à la vitesse des échanges sur les Rsn la nécessaire formation de compétences d'utilisation relativement inédites pour les individus : gestion de l'accessibilité des traces, de leur pérennité par opposition à la malléabilité des souvenirs, évaluation des contenus pertinents dans ce type de contexte aux audiences potentiellement très larges. Les techniques de soi reposent effectivement sur deux principes que Foucault appellent «la vérité locale de la sentence» et «sa valeur circonstancielle d'usage » (p. 1240) et qui sont mis à mal par l'effondrement des contextes (Boyd, 2008) et l'archivage des traces du quotidien sur les Rsn (Coutant, Stenger, 2010a).

Ce premier point permet de souligner que les Rsn constituent davantage des outils d'expression de soi que des techniques de soi.

\section{Une application absolue du principe interactionniste de la construction identitaire}

Le caractère fondamentalement co-construit de la définition de notre identité n'est plus à démontrer : «l'individu doit compter sur les autres pour compléter un portrait de lui-même qu'il n'a le droit de peindre qu'en partie » (Goffman, 1974, p. 75). Cependant, la polysémie du terme comme la difficile appréhension de ce qui lie individu et identité ont pour conséquence d'encourager encore de nombreuses visions centrées sur l'individu, qui serait alors seul décideur final des éléments identitaires qu'il diffuserait. Les techniques de soi encouragent à leur manière cette confusion puisque s'il y est question d'échange avec les autres, les cahiers employés demeurent la possession personnelle de l'individu. Ici aussi, les Rsn diffèrent largement. Le principe goffmanien y est radicalisé (Coutant, Stenger, 2010b). La construction du profil passe au moins autant par les commentaires, messages, tags, invitations déposées par les interactants, que par les activités individuelles. On en arrive même au paradoxe qu'il est relativement fréquent d'y exister sans même avoir créé de compte, les utilisateurs indiquant les participants à un événement ou taggant les personnes présentes sur une photo même si celles-ci n'ont pas créé de profil. Les techniques de soi permettent de conserver un contrôle sur les contenus partagés et diffusés que les Rsn ne permettent plus. Cette caractéristique essentielle doit être soulignée alors que beaucoup d'approches technicistes, juridiques ou sémio-quantitativistes proposent de caractériser les données personnelles en cercles 
concentriques allant du plus intime au plus public. Ainsi, elles occultent que leur définition passe par qui les produit et surtout par qui y a accès. Comprendre la forme de lien social se déployant sur ces espaces, que Perriault qualifie de «flottant» (2009), permet alors à nouveau de prendre la mesure du caractère profondément instable de la construction identitaire induite par cette logique sociotechnique.

Ajoutons que cette interactivité ne concerne pas que les interactants humains. Les outils logiciels contribuent aussi à cette perte de contrôle en participant à la construction du profil des utilisateurs par l'indication automatique de leurs activités (entrée en relation, commentaires laissés, applications installées). Objets du quotidien, les Rsn ne permettent pourtant pas à l'utilisateur une meilleure gestion de son identité pourtant traditionnellement échue à ces objets : «le maintien et la constance que l'on pense être le propre de l'individu ne sont rien d'autre que l'effet de son extériorisation et de son arrimage dans les choses familières. Les objets du quotidien ont une vertu de permanence qui construit le concret et contrôle les errements de l'identité : ils jouent le rôle de garde-fou du Soi » (Kaufmann, 2005, p. 45). Le flux n'encourage en effet en rien la stabilisation mais bien au contraire le travail sans cesse renouvelé de notre face.

Ces deux caractéristiques démontrent l'ambivalence de la fonction de support identitaire propre aux Rsn.

\section{Conclusion}

Le rapprochement avec les techniques de soi permet de formuler l'hypothèse que l'engouement pour les Rsn ne repose pas sur leurs incitations logicielles, ni sur leur réappropriation par une logique sociale, mais bien sur la capacité de leurs logiciels à intégrer dans leur fonctionnement des logiques de sociabilité fortes (Akrich, 1994). Leur succès consiste dans leur apparente proximité avec des techniques de soi développées depuis des siècles et dont les individus se révèlent particulièrement avides dans une société réservant une grande part du travail de construction de soi aux individus (Kaufmann, 2004). Cette hypothèse explique sans doute aussi les mésinterprétations sur le statut privé ou public de ces sites, envisagés à tort comme des outils personnels.

En rendant visible le quotidien, les Rsn témoignent de la diversité des petites touches constituant l'identité, souvent masquées par l'attention aux «identifiants socles » comme l'état-civil ou aux événements majeurs de la vie de l'individu (Lahire, 1998). Cependant, cette mise en visibilité ne s'accompagne pas encore de théories adaptées à la spécificité de ces 
traces et aux formes de liens flottants (Perriault, 2009) qui s'y nouent. Un champ de recherche interdisciplinaire s'ouvre donc pour saisir leur logique et permettre une meilleure formation des compétences d'utilisation de ces plateformes.

\section{Références :}

- Akrich, M., «Comment sortir de la dichotomie technique/société », in Latour, B., Lemonnier, P., De la préhistoire aux missiles balistiques : l'intelligence sociale des techniques, 1994, Paris, La Découverte, 352 p.

- Boyd D., Taken Out of Context - American Teen Sociality in Networked Publics. Doctoral Dissertation, University of California, Berkeley, School of Information, 2008, 406 p.

— Coutant, A., Stenger, T., « Pratiques et temporalités des réseaux socionumériques : logique de flux et logique d'archive », Médiation et Information, $\mathrm{n}^{\circ}$ 32, Mémoires et Internet, 2010, p. 125-136.

— Coutant, A., Stenger, T., «Processus identitaire et ordre de l'interaction sur les réseaux socionumériques », Les Enjeux de l'Information et de la Communication, août 2010, en ligne sur : http://w3.u-grenoble3.fr/les_enjeux/2010/Coutant-Stenger/index.html consulté le 13 novembre 2010.

— Foucault, M., Dits et écrits. Tome 2 : 1976-1988, Paris, Gallimard, 2001, 1736 p.

- Georges, F., Seilles, A., Artignan, G., Arnaud, B., « Sémiotique et visualisation de l'identité numérique : une étude comparée de Facebook et Myspace », 2009, en ligne sur : http://hal.archives-ouvertes.fr/docs/00/41/09/52/PDF/semiotique_finale.pdf consulté le 24 décembre 2010.

— Goffman, E., Les rites d'interaction, Paris, Les éditions de minuit, 1974, 236 p.

— Hoog, E., Mémoire, Année Zéro, Paris, Seuil, 2009, 207 p.

- Kaufmann, J-C., L'invention de soi : une théorie de l'identité, Paris, Armand Colin, 2004, $350 \mathrm{p}$.

— Kaufmann, J-C., Le cour à l'ouvrage, Théorie de l'action ménagère, Paris, Pocket, 2005, $330 \mathrm{p}$.

— Lahire, B., L'homme pluriel ; les ressorts de l'action, Paris, Nathan, 1998, 271 p.

— Martuccelli, D., Grammaire de l'individu, Paris, Gallimard, 2002, 304 p.

— Perriault J., « Traces (numériques) personnelles, incertitude et lien social », Hermès, $\mathrm{n}^{\circ} 53$, 2009, p. 13-20. 


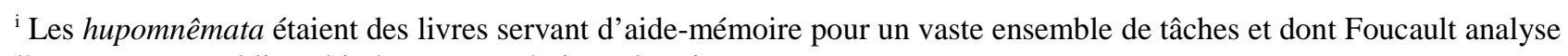
l'usage par un public cultivé comme technique de soi.

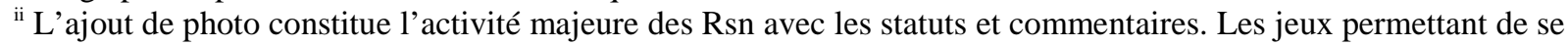
définir ou demandant aux autres de le faire y sont légions.

iii Même si celles-ci peuvent à l'occasion être réactivées par les commentaires des autres participants. 